\title{
Botryomycome : soyez prudent, n'y touchez pas
}

\section{Pyogenic granuloma, be wise, don't touch}

\section{A. Chareyron Girardot $\cdot$ O. Peyrony $\cdot$ A. Verrat $\cdot$ J.-P. Fontaine}

Reçu le 4 octobre 2013; accepté le 18 novembre 2013

(C) SFMU et Springer-Verlag France 2013

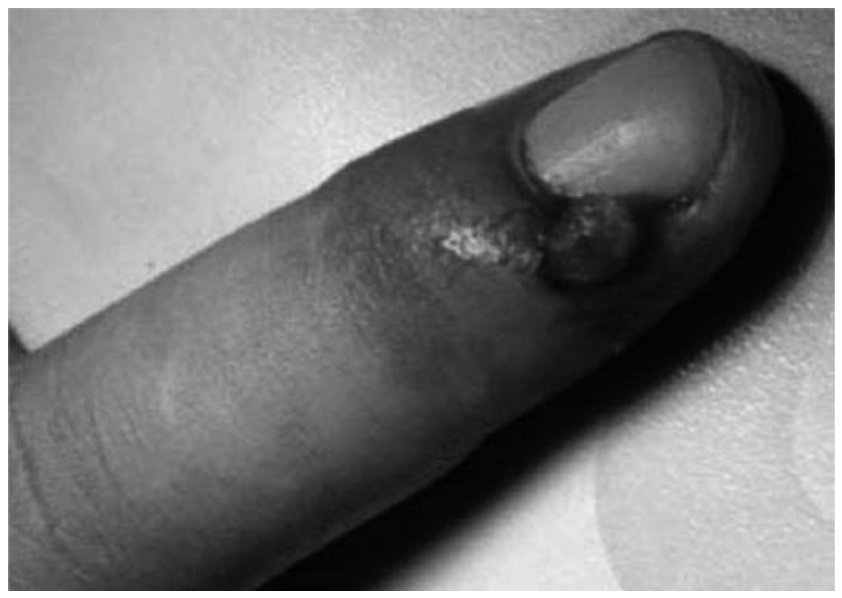

Fig. 1 Petite tuméfaction peri-ungueale vascularisée, entourée d'une collerette, évoquant un botryomycome

Un homme de 26 ans sans antécédent se présente aux urgences pour une tuméfaction péri-unguéale de l'index gauche évoluant depuis dix jours suite à une plaie. La lésion observée (Fig. 1) est en faveur d'un botryomycome. Le botryomycome, appelé aussi granulome pyogénique ou hémangiome capillaire lobulé, est une tumeur bénigne d'origine vasculaire dont la physiopathologie reste inexpliquée. Ces tumeurs sont le plus souvent de petite taille (5 à $10 \mathrm{~mm}$ ), uniques et intéressent tout le tégument cutané ou muqueux mais prédominent dans les régions acrales (péri-unguéal). $\mathrm{La}$ présence d'une collerette au pied de la lésion est fortement évocatrice et la différencie d'une collection infectieuse périunguéale (panaris). Un traumatisme cutané initial est souvent retrouvé (plaie, frottement répété, chirurgie récente). La grossesse et certains médicaments (isotretinoïne, doxetaxel, indinavir, ciclosporine ou lamivudine) favorisent l'apparition de ces lésions. Cette lésion, très vascularisée, saigne facilement au contact et récidive en cas d'exérèse incomplète [1] ; il faut donc éviter de l'inciser aux urgences. Une ablation chirurgicale sous anesthésie locale suivie d'une électrocoagulation est donc le traitement de choix pour éviter les récidives et permet, grâce à l'analyse anatomopathologique, d'éliminer un mélanome achromique [2].

\section{Références}

1. Wauters O, Sabatiello M, Nikkels-Tassoudji N, et al (2010) Le botryomycome. Ann Dermatol Venereol 137:238-42

2. Piraccini BM, Bellavista S, Misciali C, et al (2010) Periungual and subungual pyogenic granuloma. Br J Dermatol 163: 941-53

\footnotetext{
A. Chareyron Girardot $(\bowtie) \cdot$ O. Peyrony $\cdot$ A. Verrat

J.-P. Fontaine

Service des urgences, hôpital Saint-Louis,

Assistance publique-hôpitaux de Paris,

1, avenue Claude-Vellefaux, F-75010 Paris, France

e-mail : girardotchareyron@gmail.com
}

\section{A. Chareyron Girardot $\cdot$ A. Verrat}

Université Paris VII, UFR de médecine,

Site Villemin, 10, avenue de Verdun, F-75010 Paris, France 